\title{
The History of Activity of the Sun and its Rotational Period on the ZAMS
}

\author{
Kazimierz Stępień \\ Warsaw University Observatory, Al. Ujazdowskie 4, 00-478 Warszawa, \\ Poland
}

\begin{abstract}
The rotation period of the Sun after it reached ZAMS is estimated from the present rotation rate, average $\mathrm{X}$-ray emission flux and average calcium emission flux. Taking into account all existing uncertainties it is concluded that this initial period was within the range 1-9 days, with the most probable value 2-3 days. Possible influence of the solar activity on evolution of life on the Earth is briefly discussed.
\end{abstract}

\section{Introduction}

Recent observations of cool stars possessing subphotospheric convection zones suggest that stars reaching the Zero Age Main Sequence (ZAMS) rotate rapidly velocity and activity decrease continuously in time (Skumanich, 1972; Barry, 1988; Kawaler, 1988; Stępień, 1988b; Stauffer and Soderblom, 1990). It is generally accepted that the slow down of rotation is caused by the activity-related mass loss in the presence of the dynamo generated magnetic fields. The spin down decreases the efficiency of the dynamo action, which results in the weaker magnetic activity.

The loss of angular momentum can be described by (Mestel, 1984):

$$
\frac{d J}{d t} \sim \frac{d M}{d t} R_{*}^{2} \Omega\left(\frac{r_{A}}{R_{*}}\right)^{n},
$$

where $J$ is angular momentum, $d M / d t$ rate of mass loss via a stellar wind, $R_{*}$ and $\Omega$ radius and angular velocity, and $r_{A}$ the Alfvénic radius of the star. The exponent $n(0 \leq n \leq 2$ depends on geometry of the stellar magnetic field. Assuming a formula for the dependence of the stellar magnetic field on distance from the star one can replace $r_{A}$ with the surface magnetic field $B_{s u r f}$. The next step is expressing $B_{\text {surf }}$ as a function of $\Omega$. This should in principle be supplied by the dynamo theory but at present the theory is not yet enough developed. In the absence of anything better a power law has usually been adopted: $B_{\text {surf }} \sim \Omega^{p}$, with $p$ assumed (Endal and Sofia, 1981; Mestel, 1984; Kawaler, 1988; Pinsonneault et al., 
1989). Unfortunately, the usefulness of such an approximation is very doubtful. The observations of activity and surface magnetic fields indicate that for periods shorter than about 2-3 days, a saturation effect appears (Saar and Linsky, 1986; Vilhu and Walter, 1987) - contrary to an unbound increase of $B_{\text {surf }}$ with $\Omega$, predicted by the power law. A method of replacing the unknown function $B_{\text {surf }}(\Omega)$ by a semiempirical relation was suggested by Stępien $(1988 \mathrm{a}, \mathrm{b})$. The method permits also the determination of a value of the geometrical exponent from the observational data.

It assumes that the heating of stellar coronae is caused by the Alfven waves generated in tubes of the strong magnetic fields at the photospheric level. Taking into account the back-reaction of the wave generation on the generating medium a new estimate of the expected flux per unit area, $F_{A}$, was calculated. This flux can be compared with the observed X-ray flux, $F_{x}$. These two are connected by a relation: $F_{x}=f_{e} f_{A} F_{A}$, where $f_{A}$ is the filling factor of the magnetic tubes heating the corona and $f_{e}$ the efficiency factor describing all processes connected with the transmission of waves upwards, converting their energy into X-rays, geometry of the radiating region etc. Now, let us introduce a parameter $f_{x}=F_{x} / F_{A}$. Assuming that $f_{e}$ varies much less than $f_{A}$ among the discussed stars, we see that $f_{x}$ is proportional to the filling factor $f_{A}$. The exact value of the proportionality coefficient is not needed as long as we are interested only in the differential analysis.

The parameter $f_{x}$, which can also be called the (uncalibrated) filling factor, turned out to be well correlated with the directly measured surface magnetic fields for those few stars for which both quantities were known (Stępień, 1988a). This confirms the correctness of the adopted assumptions. On the other hand, $f_{x}$ has the advantage that it can be determined for each star with known $F_{x}$, i.e. for a relatively large sample of stars.

We expect that the most active stars have surface magnetic fields close to their maximum (saturation) value, hence the filling factor $f_{x}$ of these stars should be close to its maximum permissible value. Figure 2 in Stepien (1988a) shows indeed that such stars follow the line $f_{x} \approx 0.1$. The lines $f_{x}=$ const. have an inclination (Stępień, 1989b): $\log F_{x} \sim-1.43(B-V)$, or, with $\log F_{b o l} \sim 4 \log T_{e} \sim-(B-V)$ (Böhm-Vitense, 1981):

$$
\log \left(F_{x} / F_{b o l}\right) \sim-0.43(B-V) .
$$

Because these lines were determined theoretically, the resulting values of $f_{x}$ calculated for the investigated stars were called semi-empirical.

Recently Pallavicini et al. (1990) published X-ray data for a number of very active Ke and Me dwarfs. They found a tight correlation between $L_{x}$ and $L_{b o l}$ of these stars: $\log L_{x} \sim 1.21 \log L_{b o l}$. The dependence of $\log L_{b o l}$ on $(B-V)$ is given by Schmidt-Kaler (1982). In the range of spectral types K5-M6 it is approximately linear with the inclination $\log L_{b o l} \sim-2.3(B-V)$. Inserting this into the Pallavicini et al. relation we get:

$$
\log \left(L_{x} / L_{b o l}\right) \sim-0.48(B-V)
$$


in an excellent agreement with the theoretical value given by (2). Using the Pallavicini et al. relation one can calculate purely empirical filling factors from the ratio of the observed X-ray flux to its saturated value given by this relation. Due to very similar inclinations of both, theoretical and empirical, reference levels, thus determined filling factors are essentially identical with the $f_{x}$ values, apart from a constant factor.

Stępien (1988a) showed that the filling factor $f_{x}$ is well correlated with the Rossby number $R=P_{\text {rot }} / \tau_{c}$, where $P_{\text {rot }}$ is the rotation period and $\tau_{c}$ a function of the spectral type, which can be determined empirically (Stępien, 1989a), and is sometimes identified with the turnover time near the bottom of the convection zone. The resulting relation is (Stępień, 1989b): $\log f_{x}=-1.7-1.55 R$.

We can now replace $B_{s u r f}$ in the formula for loss of angular momentum with $f_{x}$ which is a known function of $R$, hence $\Omega$. After integrating the resulting equation one gets the relation $P_{\text {rot }}(t)$. The constants appearing in this relation (including the value of the exponent describing geometry of the coronal magnetic field) were determined by Stepien (1988b) from the observations of activity in open clusters of different age, obtained by Barry et al. (1987). Since that, however, several new data have appeared and it became useful to revise the calibration of $P_{\text {rot }}(t)$.

\section{The initial period of rotation of the Sun}

The relation $P_{\text {rot }}(t)$ was recalibrated in the same way as described by Stępien (1988b) but with the following modifications:

1. The Barry et al. (1987) data were reduced anew using a revised value of the basal flux given by Rutten (1987)

2. The empirical relation $\tau_{c}(B-V)$ determined by Stępien (1989a) was used to calculate the stellar Rossby numbers

3. The empirical relation between the excess calcium emission flux and $R$, found by Stẹpień (1989a) for field stars was adopted.

The new values of the calibrating constants turned out to be not much different from the old ones: $\beta=3$ (same as previously) and $T_{0}=(2.0 \pm 0.5) \times 10^{8}$, as compared to $(1.5 \pm 0.5) \times 10^{8}$ found previously.

Thus the calibrated $P_{\text {rot }}(t)$ relation permits the backward projection of the present rotation period of the Sun to its initial value on the ZAMS, or, more strictly speaking, to the value the Sun had after the phase of the possible rapid spin down suggested by observations of the youngest clusters (Stauffer and Soderblom, 1990). The result is:

$$
P_{0}(\operatorname{Sun})=2.0 \pm 0.5 \text { days, }
$$

where the uncertainty comes solely from the formal errors of the used relations.

The moderate error given above looks quite satisfactory. Unfortunately, it is definitely overoptimistic. The quoted uncertainty does not take into account all the possible systematic effects which may influence $P_{0}(\mathrm{Sun})$. Some of the important effects are: 
1. A number of $\tau_{c}(B-V)$ relations have been published (see Stępien 1989a for discussion and comparison of them). Different $\tau_{c}$ result in different relations between activity indices and the Rossby number

2. Two different measures of the activity-related calcium emission flux have been suggested in the literature (see again Stępien 1989a for discussion). It is not yet clear which one is more correct and should be used with the Barry et al. (1987) observations to calibrate the $P_{\text {rot }}(t)$ relation

3. Even when one uses the excess calcium emission flux $\Delta F_{\text {CaII }}$ (which is a better, in the opinion of the present author, measure of the chromospheric activity) one has to allow for uncertainty in the basal flux subtracted from the total measured flux to get $\Delta_{\mathrm{CaII}}$. Its value was recently revised (Rutten, 1987) but is still known with insufficient accuracy. This influences particularly strongly the determination of $\Delta F_{\mathrm{CaII}}$ for the least active stars, for which the total measured flux barely exceeds the basal flux.

The problem with calibration of the basal flux is well illustrated in Fig.1. Here the predicted excess calcium emission flux as a function of time for solar type stars with different initial periods is given as solid lines (from Stępien, 1989b). Filled circles correspond to the original data listed by Barry et al. (1987). One can notice a systematic trend between the predicted and observed fluxes, which would suggest a need for correcting the theory. Open circles correspond to the same data reduced with the new calibration. Now a trend in the opposite direction is seen. It is clear that the uncertainty of the observational data is still considerable.

Repeating the calculations of the initial period of the Sun with different relations, listed above, one gets values over a much broader range: $P_{0}(\mathrm{Sun})=1-5$ days.

The initial rotation period of the Sun can also be determined directly from the present level of the solar calcium emission (see Fig. 1). Unfortunately, the same possible systematic effects influence this method. In addition, the net calcium emission flux of the Sun is known within about $30 \%$ (judging from the values given in literature). The bars associated with the solar symbol in Fig. 1 correspond to this uncertainty. As a result, we get even broader limits: $P_{0}$ (Sun $)=0.5-9$ days.

A similar procedure can be applied to X-ray emission (see Fig. 7 in Stępień $1989 \mathrm{~b}$ ). In case of X-ray flux we have no problem with calibrating its activityrelated part - we simply assume that the whole emission is due to magnetic activity (Stępień and Ulmschneider, 1989). However, again, several diverging values of the average solar X-ray flux are given in the literature. Together with the above discussed uncertainties they lead to the following estimate: $P_{0}(\operatorname{Sun})=3-8$ days.

Summing up we conclude that the rotation period of the Sun, after it had reached ZAMS was between 1 and 9 days, with the most probable value between 2 and 3 days. The rather broad range of uncertainty is caused by a low number and a poor accuracy of the presently available observations of activity of late type stars (including the Sun itself), and an insufficient understanding of relations connecting observations and physical parameters.

In spite of the all discussed uncertainties on can attempt to reproduce, at least approximately, the evolution of rotation and activity of the Sun during its main sequence life. Figure 2 shows schematically how the rotation period, excess calcium 


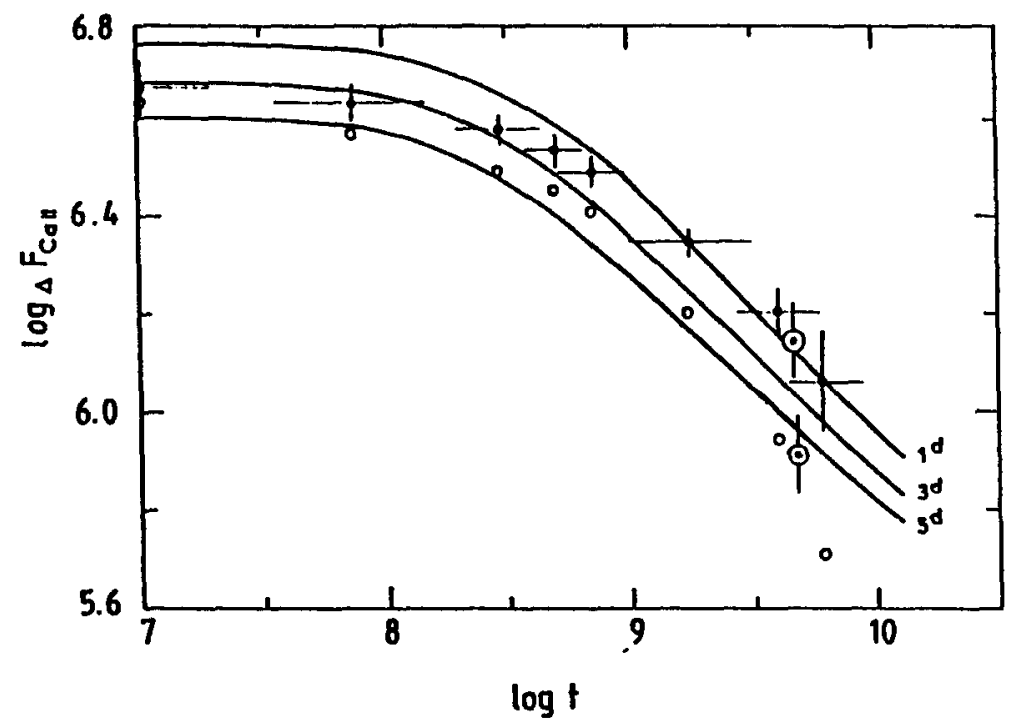

Fig. 1. The predicted calcium emission flux as a function of age for stars with different initial rotation periods (solid lines) and the observed data from Barry et al. (1987), reduced with the old (filled circles) and the new (open circles) basal flux.

emission flux and X-ray flux of the Sun varied with its age. The plotted curves are just illustrative - they do not reflect the discussed systematic effects. Nevertheless, we can draw some conclusions.

The Sun rotated on the ZAMS probably about 10 times faster than today. Its $\mathrm{X}$-ray flux was 500-1000 times larger and the chromospheric flux 4-5 times larger. It was also surely flaring very frequently and strongly. During the first billion years the Sun was losing angular momentum quite fast. As a result its period of rotation increased to a value of about 14 days, whereas the $\mathrm{X}$-ray emission decreased by a factor of about 30 . After 3.5 billion years, i.e. one billion years ago, the Sun rotated only slightly faster than presently and was nearly as active as now.

When discussing the origin and development of organic life on Earth, suggestions have been made that large amounts of ionizing radiation associated with a very high activity of the young Sun could influence the evolution of life by producing mutations at a much higher rate than presently. The above picture shows that the Sun could possibly influence in this way only the earliest forms of life existing about 3.5 billion years ago. The evolution of life during the last billion years has taken place in the presence of solar activity being essentially at the present day level.

This paper was partly supported by the grant CPBP 01.20. 


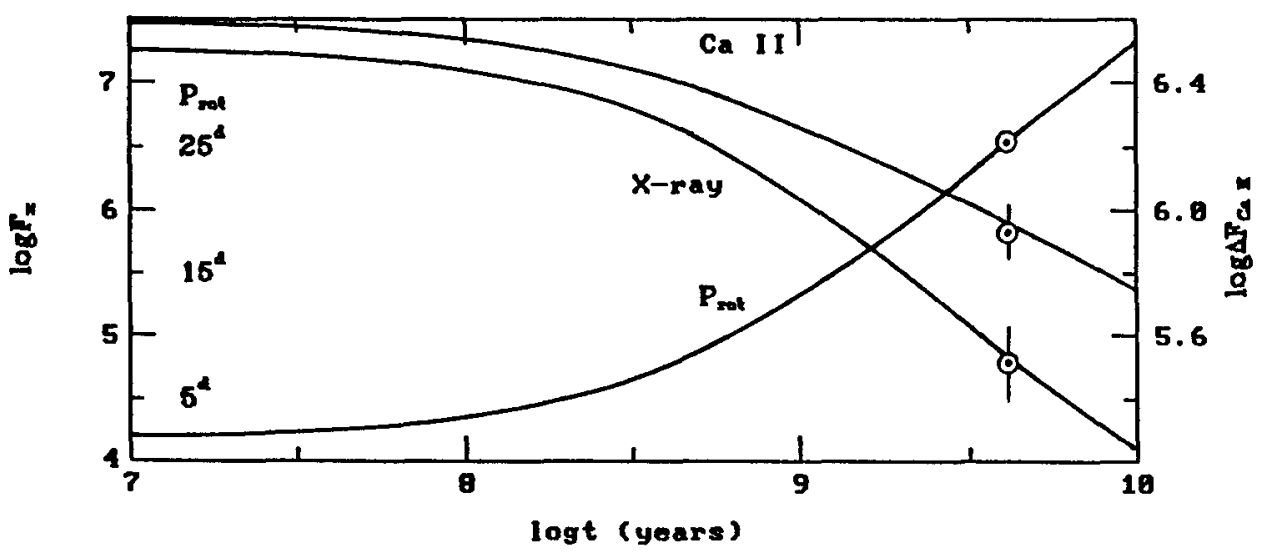

Fig. 2. The predicted evolution of rotation and activity of the Sun. Error bars correspond to uncertainties of the present levels of the solar emission.

\section{References}

Barry, D.C.: 1988, Astrophys. J. 334, 436

Barry, D.C., Cromwell, R.H., Hege, E.K.: 1987, Astrophys. J. 315264

Böhm-Vitense, E.: 1981, Ann. Rev. Astron. Astrophys. 19, 295

Endal, A., Sofia, S.: 1981, Astrophys. J. 243, 625

Kawaler, S.D.: 1988, Astrophys. J. 333, 236

Mestel, L.: 1984, in Cool Stars, Stellar Systems, and the Sun, ed. by S.L. Baliunas and

L. Hartmann, Springer, Berlin, p. 49

Pallavicini, R., Tagliaferri, G., Stella, L.: 1990, Astron. Astrophys. 228, 403

Pinsonneault, M.H., Kawaler, S.D., Sofia, S., Demarque, P.: 1989, Astrophys. J. 338, 424

Rutten, R.G.M.: 1987, Astron. Astrophys. 177, 131

Saar, H., Linsky, J.L.: 1986, Adv. Space Res. 6, 235

Schmidt-Kaler, Th.: 1982, in Landolt-Börnstein, ed. by K. Schaifers and H.H. Voigt, Springer, Berlin, Vol. 2, p. 1

Skumanich, A.: 1972, Astrophys. J. 171, 565

Stauffer, J.R., Soderblom, D.R.: 1990, Space Telescope Science Institute Preprint Series, No. 432

Stẹpień, K.: 1988a, Astrophys. J. 335, 892

Stępień, K.: 1988b, Astrophys. J. 335, 907

Stępień, K.: 1989a, Astron. Astrophys. 210, 273

Stępień, K.: 1989b, Acta Astr. 39, 209

Stępień, K., Ulmschneider, P.: 1989, Astron. Astrophys. 216, 139

Vilhu, O., Walter, F.M.: 1987, Astrophys. J. 321, 958 